\title{
Equal-Curvature X-ray Telescope Designs for Constellation-X mission
}

\author{
Timo T. Saha, David A. Content, and William W. Zhang \\ NASA/Goddard Space Flight Center, Greenbelt, MD 20771
}

\begin{abstract}
We study grazing incidence Equal-Curvature telescope designs for the Constellation-X mission. These telescopes have nearly spherical axial surfaces. The telescopes are designed so that the axial curvature is the same on the primary and secondary. The optical performance of these telescopes is for all practical purposes identical to the equivalent Wolter telescopes.
\end{abstract}

Keywords: X-Ray Optics, X-Ray Telescopes, Constellation-X

\section{INTRODUCTION}

Wolter typel telescopes have been used almost exclusively in x-ray astronomy. All the major missions are based on Wolter designs including Chandra and XMM-Newton. Hans Wolter ${ }^{1}$ introduced these designs over 50 years ago. The design consists of parabodoidal primary mirror and confocal hyperboloidal secondary mirror. The drawback of these designs is that the geometry is quite complex. The fabrication of grazing incidence conics is difficult and time consuming.

The simplest $\mathrm{x}$-ray telescope design is the cone-cone design. This design has been used successfully, for example, for BBXRT ${ }^{2}$, ASTRO-D ${ }^{3}$, and ASTRO-E ${ }^{4}$ missions. In this design the primary and secondary mirror are simple cones built from 8 quadrants. Since there is no axial curvature on the mirror surfaces, radial focusing is limited to deflection of the beam to telescope focal plane. The lag of radial focusing would consume large portion of the on-axis image requirement we have for the Spectroscopy X-ray Telescope (SXT) of the Constellation-X (CSX) mission ${ }^{5}$.

There are several complex variations of these basic designs including WolterSchwarschild designs ${ }^{6}$ and polynomial designs ${ }^{7,8,9},{ }^{10}$. They offer improvements in the resolution for narrow field applications. These designs are complex and rather difficult to manufacture to tolerances that would improve the system performance over the Wolter telescopes. Hyperboloid-hyperboloid designs ${ }^{11,12}$ offer good resolution over large fieldof-view and are used, for example, in solar applications.

Recently we introduced Equal-Curvature (EQC) grazing incidence telescopes ${ }^{13}$. The basic idea was to find simpler design alternatives for the SXT on board the CSX mission ${ }^{14}$. Thousands of segmented mirrors will be required to build the flight telescopes. This design was intended to be a simplification of the Wolter design. The EQC telescope consists of a primary mirror and a secondary mirror. The mirrors are surfaces of revolution. In the axial direction the surfaces contain polynomial terms up to second order. For small grazing angles as for CSX/SXT mirrors, the cross-sections of the 
surfaces are practically spherical and designed so that the radius of curvature is the same on the primary and secondary mirrors.

We are exploring possibilities to form and/or replicate both primary mirror and secondary mirror segments from the same mandrel. If possible, this would cut in half the mandrels needed for the fabrication of the CSX/SXT telescopes. We also believe that fabricating spherical axial profile is faster, the surfaces can be polished smoother, and the surface metrology will be easier.

In Section 2 we briefly review the design equations of the EQC telescopes. In Section 3 we describe design considerations for the CSX/SXT optic. Ray trace analysis methods we are using to compare the optical performance of the nested EQC and nested equivalent Wolter telescopes are presented in Section 4. In Section 5 we compare the optical performance of the telescopes.

\section{EQUATIONS OF EQUAL-CURVATURE TELESCOPES}

The simplest design after the cone-cone telescope is a design with spherical crosssections. The spherical cross-sections will provide radial focusing and improve the onaxis imaging capability of the telescope. The spherical cross-section of the primary $(j=1)$ and secondary $(j=2)$ can be expressed as:

$$
h_{j}=h_{0}-R \cos \left(i_{j 0}\right) z_{j}+\left[R^{2}-\left(z_{j}+R \sin \left(i_{j 0}\right)\right)^{2}\right]^{1 / 2} \text {, }
$$

where $h_{0}$ is the radial height at the surface intersection, $i_{j 0}$ is the slope angle of the primary or secondary at the surface intersection point, and $R$ is the radius of curvature of the mirrors. Expanding the square root of Eq.(1) and keeping only terms up to second order, we get:

$$
h_{j}=h_{0}-R \cos \left(i_{j 0}\right) z_{j}-\frac{z_{j}{ }^{2}}{2 R \cos \left(i_{j 0}\right)^{3}}=a_{j 0}+a_{1 j} z_{j}+a_{2 j} c z_{j}{ }^{2} \text {, }
$$

where $\mathrm{c}$ is the curvature of the surfaces $(=1 / R)$ and $a_{j 0}, a_{1 j}$, and $a_{2 j}$ are constants. The higher order terms in the expansion are negligible as long as the radius of curvature of the mirrors is much larger than the axial coordinate $z_{j}$.

Equations (2) are the base for the EQC telescopes. Dropping the second order term would reduce the design to the cone-cone telescope. The cross-section of the telescope and definition of the basic parameters are shown in Figure 1.

It can be shown ${ }^{13}$ that the optimum on-axis imaging is achieved if the curvature of the mirrors is:

$$
c=\frac{i_{10}-(49 / 6) i_{10}{ }^{3}}{4 L}+\frac{3 i_{10}\left[\left(L_{1} / 2\right)+c_{1}\right]}{16 L^{2}},
$$

where $L$ is the axial distance from the surface intersection plane to the focus of the telescope, $L_{l}$ is the axial length of the primary mirror, and $c_{l}$ is the axial distance from the end of the primary to the surface intersection plane. Eq. (3) is an approximation. Only 
low order terms in $1 / L$ and $i_{10}$ are included. The radial height at the surface intersection is then:

$$
h_{0}=L \tan \left(4 i_{10}\right)+z_{11}\left[i_{10}+\frac{25}{3} i_{10}{ }^{3}-c L\left(4+66 i_{10}{ }^{2}\right)\right]+c z_{11}{ }^{2}\left(\frac{1}{2}-\frac{8 c L}{i_{10}}\right) \text {, }
$$

where $z_{I I}=-L_{1} / 4-c_{1}$.

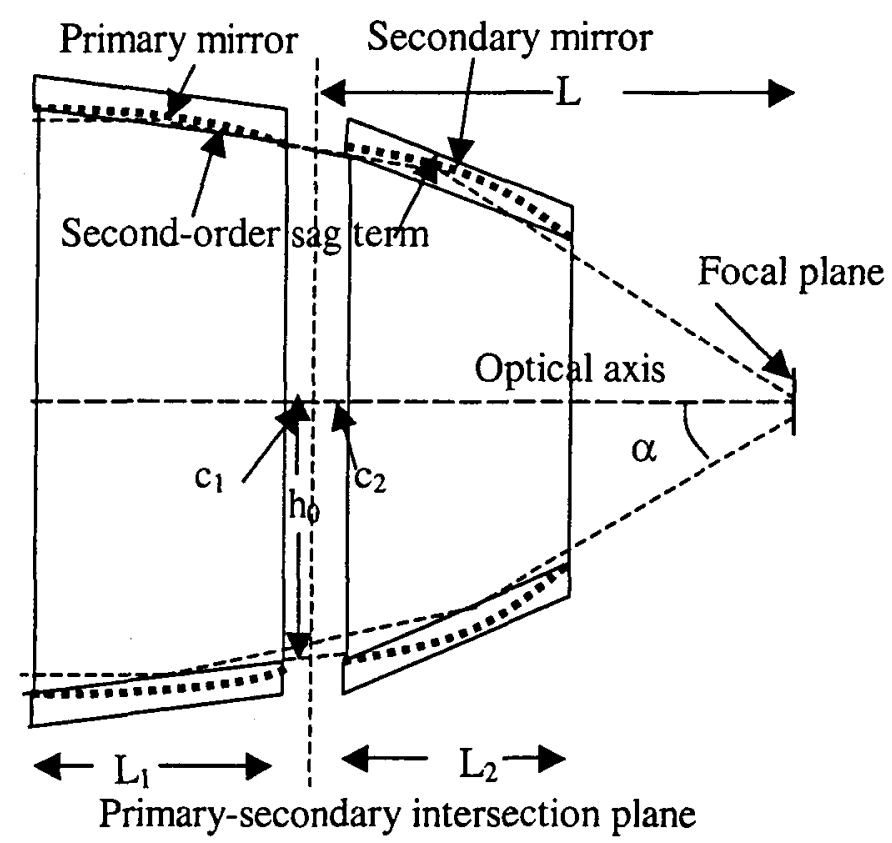

Figure 1. Schematic drawing of the telescope. Thick dashed lines represent the surfaces of EQC telescope.

\section{CSX/STX TELESCOPE DESIGNS}

Dimensional, field-of-view, and performance requirements of the CSX/SXT telescopes are shown in Table 1. These requirements define the design envelope. We have two design concepts: 1) axial length of the mirrors $300 \mathrm{~mm}$ and 2) the axial length of the mirrors $200 \mathrm{~mm}$. The mirrors are separated by a cap that is $50 \mathrm{~mm}$ long. The cap between the end of the primary and the intersection plane is $25.1 \mathrm{~mm}$ and the cap from the intersection plane to the front of the secondary is $24.9 \mathrm{~mm}$. We assume the mirror thickness of $0.44 \mathrm{~mm}$ and add additional $0.2 \mathrm{~mm}$ space between the shells to allow room for alignment errors for the mirror segments. The maximum and minimum radial heights can vary from $800 \mathrm{~mm}$ to $150 \mathrm{~mm}$.

\section{Table 1.CSX/SXT telescope requirements}

Number of mirror systems

Mirror radial diameter

Mirror focal length

Telescope angular resolution

Mirror axial length

Telescope field-of-view

\section{4}

$1.6-0.3 \mathrm{~m}$

$10 \mathrm{~m}$

15 arcsec (goal arcsec)

$200-300 \mathrm{~mm}$

2.5 arcminutes 
Under these conditions we can fit 167 shells that are $300 \mathrm{~mm}$ long and 230 shells that are $200 \mathrm{~mm}$ long within the radial limits.

\section{OPTICAL PERFORMANCE MODEL}

We use the EE version of the ZEMAX ${ }^{15}$ optical design program to analyze the CSX/SXT Wolter and EQC telescopes. The nested surfaces of the CSX/SXT telescope are setup as non-sequential components (NSC) in ZEMAX. The large amount of surfaces makes it impractical to enter all the surfaces by hand. Therefore, we wrote an IDL program that automatically generates the ZEMAX input file from the design parameters.

We used the 'standard surface' object to describe the surfaces of the Wolter telescope in ZEMAX. It can be shown that the radius of curvature of the primary $\left(R_{l}\right)$, radius of curvature of the secondary $\left(R_{2}\right)$, and eccentricity of the secondary $(\varepsilon)$ of the Wolter telescopes can be calculated from the radial height at the primary-secondary intersection plane $\left(h_{0}\right)$ and the axial distance from the intersection plane to the focus $(L)$. The equations are:

$$
\begin{aligned}
& \alpha=a \tan \left(h_{0} / L\right) \\
& R_{1}=-h_{0} \tan (\alpha / 4) \\
& \varepsilon=1 /(2 \cos (\alpha / 2)-1) \\
& R_{2}=\varepsilon R_{1}
\end{aligned}
$$

These equations are valid only if the grazing angles of the primary mirror and secondary mirror are the same at the primary-secondary intersection point. All of the ZEMAX input parameters can be easily calculated from these equations.

The surfaces of the EQC telescopes are more difficult to setup in ZEMAX. We use an object called 'an aspheric surface' to describe the EQC telescopes. The surface equation of this object surface is:

$$
z_{j}=\frac{c u r v_{j} h_{j}{ }^{2}}{1+\sqrt{1-\left(1+k_{j}\right) c u r v_{j}{ }^{2} h_{j}{ }^{2}}}+\sum_{i=1}^{N} \alpha_{i j} h^{i}
$$

where $c u r v_{j}$ is the curvature of the surface, $k_{j}$ is the conic constant of the surface, and $\alpha_{j}$ are the coefficients of the polynomial terms. To relate the parameters of equation (9) to the parameters of Eq. (2), Eq.(2) is solved for $z_{j}$ and expanded. For the ZEMAX thickness parameters and the parameters of Eq.(9) we find:

$$
\begin{aligned}
& \text { thickness }_{j}=-h_{0} / a_{1 j}-a_{2 j} c_{0}{ }^{2} / a_{1 j}{ }^{3}-2\left(a_{2 j} c\right)^{2} h_{0}{ }^{3} / a_{1 j}{ }^{5}-5\left(a_{2 j} c\right)^{3} h_{0}{ }^{4} / a_{1 j}{ }^{7} \\
& \alpha_{1 j}=1 / a_{1 j}+2\left(a_{2 j} c\right) h_{0} / a_{1 j}{ }^{3}+6\left(a_{2 j} c\right)^{2} h_{0}{ }^{2} / a_{1 j}{ }^{3}+20\left(a_{2 j} c\right)^{3} h_{0}{ }^{3} / a_{1 j}{ }^{7} \\
& \text { curv }_{j}=-a_{2} c / a_{1}{ }^{3}-6\left(a_{2} c\right)^{2} h_{0} / a_{1}{ }^{5}-30\left(a_{2} c\right)^{3} h_{0}{ }^{2} / a_{1}{ }^{7} \\
& \alpha_{2 j}=0
\end{aligned}
$$




$$
\begin{aligned}
& \alpha_{3 j}=2\left(a_{2 j} c\right)^{2} / a_{1}{ }^{5}+20\left(a_{2} c\right)^{3} h_{0} / a_{1 j}{ }^{7} \\
& \alpha_{4 j}=-5\left(a_{2} c\right)^{3} / a_{1 j}{ }^{7} .
\end{aligned}
$$

Terms higher than $4^{\text {th }}$ order in radial height are negligible for the CSX/SXT telescope.

The entrance aperture of the telescope consists of large number of narrow annuli. A large portion of the rays misses the surfaces if we use the default ZEMAX ray sets. Therefore, we decided to write a macro that includes only the random set of rays that actually enter the telescope through the entrance aperture. We used the POLTRACE command of ZEMAX to ray trace the optical system. The macro also saves the ray intercepts and intensities of the rays at the image plane in a file. We wrote an IDL program to calculate the image centroids and RMS spot diameters from the ZEMAX ray trace data.

We assumed a gold coating on the surfaces and used wavelength of $1.0 \mathrm{KeV}$ in the ray $r$ trace analysis.

\section{RESULTS OF RAY TRACE ANALYSIS}

A cross-section of the ZEMAX 3D layout drawing for the nested Wolter telescope is shown in Figure 2. The mirrors are $300 \mathrm{~mm}$ long and there are 167 shells nested inside each other.

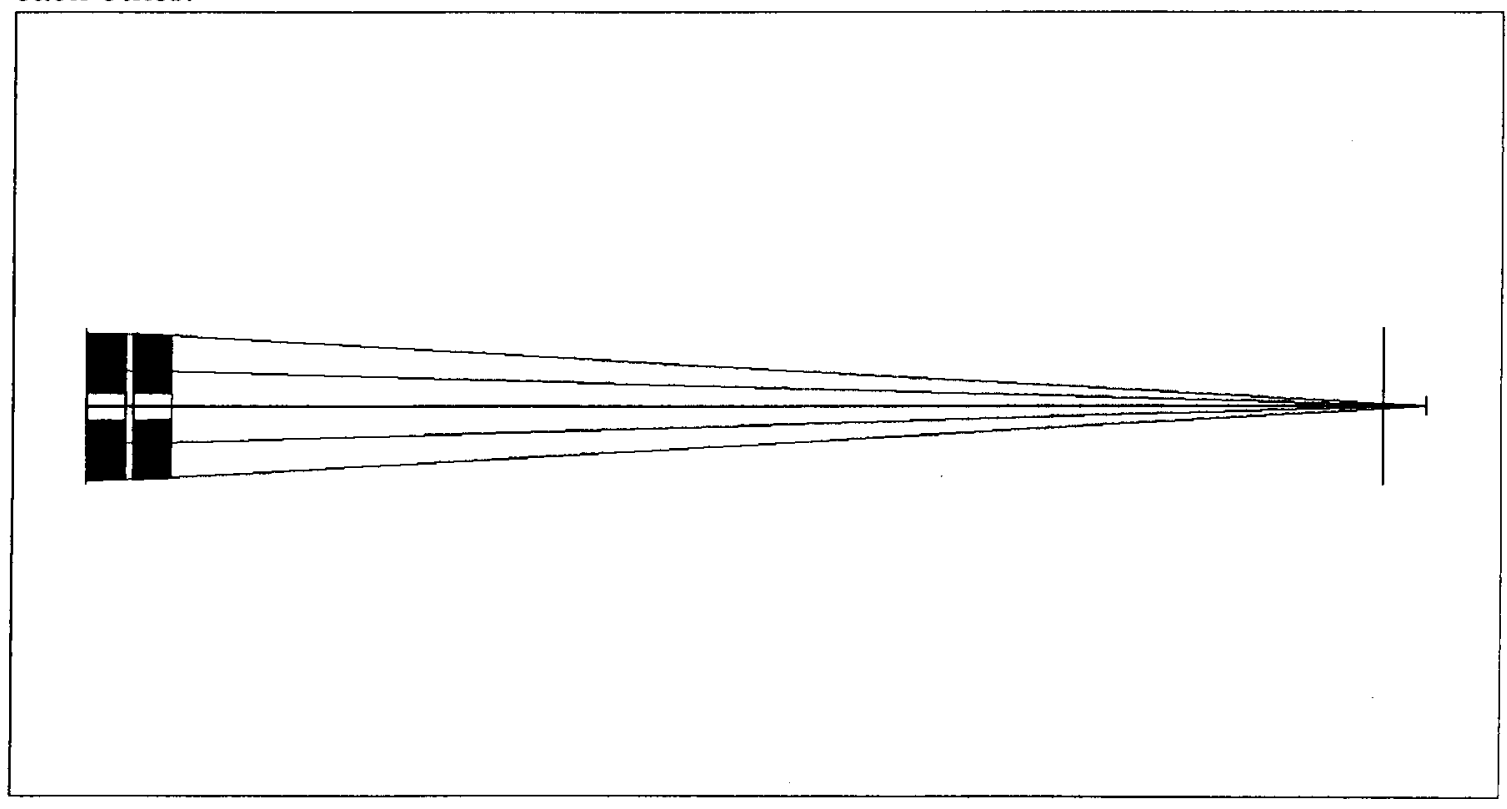

Figure 2. ZEMAX layout drawing of the nested 167 shell CSX/SXT telescope. The mirrors are 300 $\mathrm{mm}$ long. The cap between the primary and secondary is $50 \mathrm{~mm}$ and the distance from the entrance aperture to the telescope focus is $\mathbf{1 0 3 2 5 . 1} \mathrm{mm}$.

The ray trace results for the Wolter and EQC telescopes are shown in Figure 3 and 4. In Figure 3, the RMS spot diameter is plotted as a function of the half-field angle for the 200-mm design. The spot diameter of the EQC design is slightly larger at small field angles. The on-axis RMS spot diameter of the EQC design is only about 0.05 arcsec. After about HFA of 1 arcmin the RMS spot diameters of the two designs are nearly identical. 


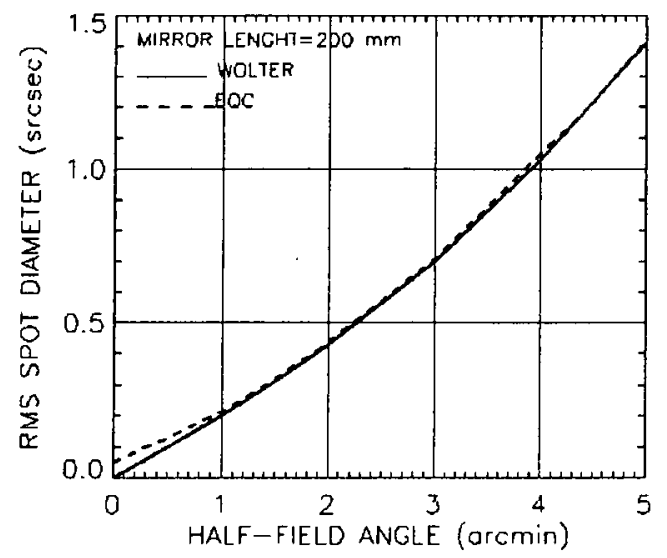

Figure 3. The RMS blur circle diameter as a function of the half-field angle for nested Wolter and EQC designs at $1 \mathrm{KeV}$. The mirrors are $200 \mathrm{~mm}$ long.

The RMS spot diameter of EQC and Wolter designs with 300-mm long mirrors is shown in Figure 4. In this case the RMS spot diameter of the Wolter telescope is slightly smaller across the 5-arcmin field-of-view. The on-axis RMS spot diameter of the EQC design is about 0.1 arcsec.

The science FOV diameter of the CSX/SXT telescope is 2.5 arcmin. Within this FOV the on-axis and off-axis RMS blur diameters are miniscule compared to imaging errors allocated for manufacturing, alignment, and other error sources. The optical performance requirement is 15 arcsec half power diameter (HPD).

The shorter telescope has slightly better on- and off-axis performance. The RMS spot diameter at the edge of 10 arcmin FOV increases from 1.4 arcsec to 1.8 arcsec when the mirror length is increased from $200 \mathrm{~mm}$ to $300 \mathrm{~mm}$. At the edge of the CSX science FOV the RMS spot diameters are 0.26 arcsec for the short design and 0.32 arcsec for the long design.

\section{SUMMARY}

The optical performance of the nested EQC telescopes is nearly identical with similarly sized nested Wolter telescopes. The on-axis RMS spot diameter of the studied designs is about 0.1 arcsec or smaller. The increased image blur is miniscule compared to the telescope optical performance requirement $(\mathrm{HPD}=15$ arcsec).

The primary and secondary of the EQC telescopes have the same axial curvature. We plan to take advantage of this feature. We are investigating options of forming and/or replicating primary mirror segments and secondary mirror segments from a single 